\title{
Elucidation of the Photoreactivity of nanocrystalline 2-hydroxychalcones using continuous flow method
}

\author{
Sobiya George, ${ }^{\dagger}$ Jeanette A. Krause, ${ }^{\dagger}$ and Anna D. Gudmundsdottir*, ${ }^{\dagger}$ \\ ${ }^{+}$Department of Chemistry, University of Cincinnati, Cincinnati, Ohio 45221-0172, United States
}

\begin{abstract}
The 2-hydroxychalcones form an important group of organic compounds not only because of their pharmacological properties but also because they are intermediates in the biosynthesis of flavanones. We studied the photoreactivity of 2-hydroxychalcone derivatives and found that their photochemistry is both wavelength and concentration dependent. Irradiation of 2- hydroxychalcone derivatives with $365 \mathrm{~nm}$ LED in dilute concentration selectively forms flavanones, whereas at higher concentrations an additional photoproduct is observed. Irradiation of nanocrystalline 2-hydroxychalcone may result in higher selectivity since the concentration will not be a limiting factor in solid state. To explore the photoreactivity of nanocrystalline 2-hydroxychalcones we will be using the continuous flow method. The wavelength specific and concentration dependent photoreactivity of 2-hydroxychalcones is explained using trapping studies, laser flash photolysis and TD-DFT calculations.
\end{abstract}

